\title{
The renin - angiotensine system and psychiatric disorders in pregnancy and post - partum
}

\author{
Roxana Mihaela Barbu, Cristina Maria Gavrilescu, Elena Cojocaru, \\ Crînguța Paraschiv, Ionuț R. Popescu, Walther Bild
}

\begin{abstract}
Roxana Mihaela Barbu - MD, PhD, University of Medicine and Pharmacy „Grigore T. Popa”, Iași

Cristina Maria Gavrilescu - MD, PhD, University of Medicine and Pharmacy „Grigore T. Popa”, Iași

Elena Cojocaru - MD, PhD, University of Medicine and Pharmacy „Grigore T. Popa”, Iași Crînguța Paraschiv - MD, PhD, University of Medicine and Pharmacy „Grigore T. Popa”, Iași Ionuț R. Popescu - MD, PhD, University of Medicine and Pharmacy „Grigore T. Popa”, Iași Walther Bild - MD, PhD, professor, University of Medicine and Pharmacy „Grigore T. Popa”, Iași
\end{abstract}

\begin{abstract}
The renin-angiotensin system is one of the most important general and local hormonal systems in the body. Circulating angiotensinogen, under the action of renal or extrarenal renin is transformed into angiotensin $I$. Angiotensin $I$ is a nonapeptide, which is not active per se but by transforming into angiotensin II and by stimulating the secretion of aldosterone. The AT2 receptors are present mainly in the uterus, adrenal gland, but also in the fetus, neonate and in the brain. The renin-angiotensin system is an endocrine system of major importance in physiology and physiopathology, with huge implications in different pathologies, including psychiatric ones. Psychiatric diseases in the perinatal period are a public health problem because of the prevalence, the relative ignorance on this type of pathology and because of the double effect on the mother and on the fetus. Perinatal psychopathology studies the psychological difficulties and psychiatric disorders that can occur during pregnancy and postpartum. It has consequences on the early mother-child relationship as well as the psychotropic treatments that can be given during pregnancy and lactation and their short, medium and long term effect on the newborn, as well as the risks that may occur when treatment is stopped abruptly at the beginning of pregnancy. Conclusions: Considering that the status and type of the myometer angiotensin receptors is very unclear and little studied and that hormonal changes are the main cause for both baby blues and postnatal depression, further physiological, physiopathological and clinical studies are needed to thoroughly assess the involvement of renin-angyotensine-aldosteron system in the psychiatric disorders in pregnancy and postpartum.
\end{abstract}




\section{INTRODUCTION}

The renin-angiotensinsystem (RAS), or reninangiotensin-aldosterone system (RAAS) is a hormonal cascade that functions in the homeostatic control of blood pressure, tissue perfusion and extracellular volume. Circulating angiotensinogen, under the action of renal or extrarenal renin is transformed into angiotensin I. Angiotensin I is a nonapeptide, which is not active per se but by transforming into angiotensin II or stimulating the secretion of aldosterone. The renin-aldosterone system controls sodium and potassium balance, blood volume and blood pressure. Hypovolemia, hyponatremia and other causes of low blood pressure stimulate the secretion of renin and increase aldosterone levels. Aldosterone causes hydrosaline retention, increases blood pressure and loss of potassium. Assessing renin is useful in the different diagnosis of hypertensive patients. The renin-angiotensin system is involved in maintaining the electrolyte balance in the body and thus in controlling blood pressure. The angiotensine II (AT 2) receptors are a class of proteincoupled receptors with angiotensin II; they are resposible for the signal transduction of the vasoconstricting stimulus of angiotensin II. The AT2 receptors are present mainly in the uterus, adrenal gland, but also in the fetus, neonate and in the brain. Drugs that inhibit RAAS are used especially in the treatment of high blood pressure and congestive heart failure, while some drugs are also used in certain kidney diseases to reduce proteinuria $(1,3)$.

\section{THE RENIN - ANGIOTENSIN - ALDOSTERONE SYSTEM IN PREECLAMPSIA}

Preeclampsia is physiologically characterized by plasma volume contraction, intravascular coagulation and intense vasoconstriction. It was initially thought that the reninangiotensin-aldosterone system would be hyperactive, but studies have shown a more complex picture. Plasma renin activity and concentration, in addition, and plasma concentrations of angiotensin II (ANG II) and aldosterone are reduced compared to normal pregnancy. The total concentration of renin is normal, and plasma concentrations of high molecular weight angiotensinogen are increased in preeclampsia, although total angiotensinogen is normal. Plasma renin activity and concentration respond appropriately to physiological stimuli in preeclampsia, except for the release of impaired renin after furosemide, possibly due to prostacycline deficiency. Although the plasma concentrations of angiotensin II are low, there is an increased pressure sensitivity to infused ANG II with unknown mechanisms. The plasma concentration of aldosterone is low, but the ratio of aldosterone concentration to plasma renin concentration is twice as high in preeclampsia than normal pregnancy. This does not appear to be due to changes in potassium, atrial natriuretic peptide, dopamine or ACTH but may be another manifestation of increased (adrenal) sensitivity to ANG II in preeclampsia (2).

There is an inverse relationship between the ratio of active renin to prorenin and the clinical severity of preeclampsia. Understanding the mechanisms that produce these changes in the RAA system in preeclampsia will give strong clues to the general pathogenesis of this disorder. The onset is before, during or after birth. The seizures are of the tonic-clonic type and usually last about a minute. As a result of the crisis, there is usually a period of confusion or coma. Complications of preeclampsia include aspiration pneumonia, cerebral hemorrhage, renal failure, and cardiac arrest $(3,5)$.

Eclampsia is a severe condition and is the last phase of preeclampsia. The pregnant can suffer from epileptic seizures, seizures and loss of consciousness, which can lead to coma or loss of mother and baby's life. Preeclampsia and eclampsia are part of a larger group of disorders known as hypertensive pregnancy disorders (6). 


\section{POST-PARTUM PSYCHIATRIC DISORDERS}

Pregnancy, but especially puerperality, are periods of vulnerability to the appearance or recurrence of psychiatric disorders, the changes due to some: psychic factors: interpersonal and intrapsychic changes; biological factors: hormonal changes and social factors. Pregnancy protects against serious psychiatric episodes, but the psychiatric symptoms that occur during this period are more frequent and more intense than among the general population. Leukemia is a period of increased risk for delusions or depressions often unknown before, with direct effects on the child. Perinatal psychiatry is a border discipline between pediatric psychiatry, adult psychiatry, obstetrics and pediatrics. It aims to address several psychological problems: mental illness of parents, early interactive dysfunctions, training and awareness. It has both a preventive role in identifying the high-risk psychiatric population and in preventing attachment disorders as well as a curative role (10).

Besides the fact that the postpartum period comes after the last trimester, with a lot of fatigue and lack of sleep, combined with the stress of birth, immediately after the expulsion of the placenta, abrupt decreases in hormonal levels during pregnancy occur. Most women, up to $80 \%$, may have periods of marked emotionality, unexplained tears, fears, and may feel painfully a sense of rupture between mother and newborn baby, who suddenly demands the right to a separate existence. of the mother's body (12).

Postpartum sadness, also called baby blues, is normal after birth and affects about $70 \%$ of new moms. The culprits are the radical changes that take place in the mother's life with the birth of the baby, but also the hormonal disorders that occur during the first weeks. Symptoms usually appear within a few days after birth and gradually disappear after the first few weeks. Baby blues are manifested by sudden mood swings, sadness, anxiety, confusion, irritability, difficulties in resting even if the child is asleep, lack of energy, appetite or concentration problems. If the sadness persists even after a maximum of one month, or if the symptoms do not improve, but even worsen, postnatal depression occurs (11).

Unlike baby blues, postpartum depression is a more serious condition, having about the same symptoms, except that these symptoms not only do not disappear after a few weeks after birth, but often worsen. Sometimes, the symptoms become more distressing, the suffering is greater and the life of the fresh mother is significantly affected with a frequency of up to $20 \%$ of women.

A postpartum depressive episode occurs within the first four weeks after birth, and includes several symptoms that are severe enough to affect daily life: sadness, depressive mood; inability to enjoying oneself; feelings of guilt for these states or for other things; fear, anxiety, exaggerated fear of hurting the child; the feeling of being overtaken by events; sleep problems caused by mental distress; crying accesses; the idea of harming themselves, headaches, tingling or numbness of the limbs. Chest pain and palpitations may also be symptoms of postpartum depression. Some mothers who suffer from postpartum depression develop new phobias, panic attacks or nightmares. The feeling of guilt goes often together with that of inadequacy and hostility both to oneself or to the others. Severe concentration or memory problems also occur. In more severe cases, the mother may even have negative child-oriented thoughts or suicidal thoughts. Although it usually appears after birth and progressively progresses, postpartum depression can also develop suddenly, anytime in the first year of life of the child. Statistics show that about $10 \%$ of mothers suffer from postnatal depression (12).

The status and type of myiometer angiotensin receptors have always been very unclear and little studied. A report from 1996, using human myometrial fragments, observed a number of distinct features, both at the level of the uterine smooth muscle per se and at the level of the smooth vascular musculature in 
the uterine wall. Angiotensin II significantly increases muscle contractility stimulated by electric field, and by stimulating angiotensin receptors increases the amount of calcium available in smooth muscle cells, which amplifies the effect of electrical stimulation (7).

Blocking studies have shown that in the female uterine musculature binding is almost exclusively to AT2 receptors, sensitive to PD123177, and very little to AT1 receptors (losartan blockers). Also, the vascular myometrium has shown a clear preference for binding, significantly stronger for angiotensin II, compared with the myometer with dissected vessels, which shows that the essential presence of the receptors is especially at the level of the vascular structures $(8,14)$.

Another major finding is that the expression of AT2 receptors was higher during the follicular phase than during the luteal phase, which allows greater prostacycline synthesis. Comparative studies on human and murine cell lines have shown that PD123319 (a selective AT2 antagonist) inhibited radioactive iodine-labeled Ag coupling, while losartan did not influence this coupling, neither on human nor on murine preparations (9).

Hormonal changes are the main cause for both baby blues and postnatal depression. If during pregnancy hormone levels such as estrogen and progesterone are extremely high, after birth they drop sharply, leading to sadness or even depression. In addition, thyroid hormone levels may drop significantly after birth, and thyroid deficiency can lead to an increased state of fatigue and depression. Stress is another important factor in triggering postpartum depression, along with lack of sleep, new responsibilities that the mother has and fear of the unknown. Physical changes may be another cause of postpartum depression (11).

The treatment of postpartum depression consisted of antidepressant medication and hormone therapy, respectively estrogen therapy. Estrogen treatment for postpartum depression has been poorly studied. Some women taking estrogens have shown improvements in their health, however some of them have had to take an antidepressant at the same time, so it is hard to know if the effect was due exclusively to estrogen. However, estrogen therapy is unlikely to become a common treatment for postpartum depression because it increases the risk of deep vein thrombosis and endometrial cancer. Concomitant addition of progesterone reduces the risk of endometrial cancer of estrogen, but is known to be a trigger for postpartum depression, if given after birth. Statistics show that about $25 \%$ of mothers who do not receive treatment for postpartum depression continue to suffer from depression even one year after birth(14).

\section{CONCLUSIONS}

Considering that the status and type of the myometer angiotensin receptors is very unclear and little studied and that hormonal changes are the main cause for both baby blues and postnatal depression, further physiological, physiopathological and clinical studies are needed to thoroughly assess the involvement of renin-angyotensine-aldosteron system in the psychiatric disorders in pregnancy and postpartum.

\section{ACKNOWLEDGEMENTS AND DISCLOSURES}

The authors declare that they have no potential conflicts of interest to disclose 


\section{REFERENCES}

1. Abdalla S, Lother H, Abdel-tawab AM et al. The angiotensin II AT2 receptor is an AT1 receptor antagonist. J Biol Chem, 2001; 43: 39721-6.

2. Accorsi-Mendonça D, Corrêa FM, Anselmo-Franci JA, Paiva TB, de Oliveira AM. Angiotensin actions on the isolated rat uterus during the estrous cycle: influence of resting membrane potential and uterine morphology. Pharmacology, 2002; 65: 162-169.

3. Brailoiu E, Churamani D, Pandey V, Brailoiu GC, Tuluc F, Patel S, Dun NJ (2006). Messenger-specific role for nicotinic acid adenine dinucleotide phosphate in neuronal differentiation. J Biol Chem 281: 15923-15928.

4. Gard PR, Daw P, Mashhour ZS, Tran P. Interactions of angiotensin IV and oxytocin on behaviour in mice. J Renin Angiotensin Ald Syst, 2007; 8: 133-138.

5. Hannan RE, Gaspary TA, Davis E, Widdop RE. Differential regulation by AT1 and AT2 receptors of angiotensin II-stimulated cyclic GMP production in rat uterine artery and aorta. BJ Pharmacol, 2004; vol 141 (6): 1024-31.

6. Nagata S, Kato J, Sasaki K, Minamino N, Eto T, Kitamura K. Isolation and identification of proangiotensin-12, a possible component of the renin-angiotensin system. Biochem Biophys Res Commun, 2006; 350: 1026-1031.

7. Neves LA, Stovall K, Joyner J, Valdés G, Gallagher PE, Ferrario CM, Merrill DC, Brosnihan KB. ACE2 and ANG- (1-7) in the rat uterus during early and late gestation. Am J Physiol Regul Integr Comp Physiol, 2008; 294 :

R151-161.

8. Schrlau MG, Brailoiu, Patel S, Gogotsi Y, Dun NJ, Bau HH (2008). Carbon nanopipettes characterize calcium release pathways in breast cancer cells. Nanotechnology 19: 325102.

9. Varagic J, Trask AJ, Jessup JA, Chappell MC, Ferrario CM. New angiotensins. J Mol Med, 2008; 86: 663-671.

10. Vancea, Florin, Elements of psychopathology and psychiatry, Psychomedia Ed. , Sibiu, 2008.

11. Vancea, Florin, The Basics of Psychopathology, Psychomedia Ed., Sibiu , 2011.

12. Tudose, F, Psychopathology and psychiatry for psychologists, Infomedica Ed. , Buc ., 2002.

13. Widdop RE, Matrougui K, Levy BI, Henrion D. AT2 receptor-mediated relaxation is preserved after long-term AT1 receptor blockade. Hypertension. 2002; 40: 516-520.

14. Wray S, Kupittayanant S, Shmygol A, Smith RD, Burdyga T (2001). The physiological basis of uterine contractility: a brief review. Exp Physiol 86: 239-46.

\section{Correspondence:}

Cristina Gavrilescu,

MD, PhD, University of Medicine and Pharmacy „Grigore T. Popa”, Iasi, Universitatii street no 16, 700115, Iași, Romania, cristina.gavrilescu@umfiasi.ro

Submission:14sep2019

Acceptance: 18nov2019 